\title{
Tumoral PD-L1 expression in desmoplastic melanoma is associated with depth of invasion, tumor-infiltrating CD8 cytotoxic lymphocytes and the mixed cytomorphological variant
}

Noah Frydenlund ${ }^{1}$, Dominick Leone ${ }^{2}$, Shi Yang ${ }^{3}$, Mai P Hoang ${ }^{4}$, April Deng ${ }^{5}$, Marier Hernandez-Perez ${ }^{6}$, Rajendra Singh ${ }^{7}$, Asok Biswas ${ }^{8}$, Ron Yaar $^{9}$ and Meera Mahalingam ${ }^{10}$

${ }^{1}$ Unversity of Iowa Carver College of Medicine, Iowa City, IA, USA; ${ }^{2}$ Boston University School of Public Health and Ragon Institute of MGH, MIT and Harvard, Boston, MA, USA; ${ }^{3}$ Department of Pathology, Boston University School of Medicine, Boston, MA, USA; ${ }^{4}$ Department of Pathology, Massachusetts General Hospital, Boston, MA, USA; ${ }^{5}$ Department of Pathology, University of Massachusetts Medical School, Worcester, MA, USA; ${ }^{6}$ Miraca Life Sciences, Newton Upper Falls, MA, USA; ${ }^{7}$ Department of Pathology, Icahn School of Medicine at Mount Sinai, New York, NY, USA; ${ }^{8}$ Department of Pathology, Western General Hospital, Edinburgh, Scotland; ${ }^{9}$ Aurora Diagnostics GPA Laboratories, Greensboro, NC, USA and ${ }^{10}$ Dermatopathology Section, Department of Pathology and Laboratory Medicine (113), VA Integrated Service Networks (VISN1), West Roxbury, MA, USA

Recently, patients with metastatic desmoplastic melanoma (DM) have been shown to respond more favorably to anti-PD1/PD-L1 therapy than other melanoma subtypes. Given this, we evaluated PD-L1/2 expression in primary DM samples and correlated these with subtype, CD8+ lymphocyte status, histopathological prognosticators, and select genetic alterations. Eighty-six (36 mixed DM, 50 pure DM) archival annotated samples met inclusion criteria and were immunohistochemically semiquantitatively evaluated. Per established criteria, for PD-L1/L2, cases with $\geqslant 5 \%$ tumoral expression, and for CD8, cases with a predominantly peri/intratumoral CD8+ infiltrate were scored positive. Univariate analysis (chi-square and Wilcoxon) identified potential confounders and a nested case-control study was accomplished using multiple logistic regression. For PD-L1, $49 \%$ of cases were positive and $71 \%$ of cases with thickness $>4 \mathrm{~mm}$ were positive; PD-L1 expression differed by median depth ( $3.29 \mathrm{~mm}$, interquartile range $=\mathbf{3 . 5 8} \mathrm{mm}$ for PD-L1 positives vs $1.75 \mathrm{~mm}$, interquartile range $=\mathbf{2 . 0 4} \mathrm{mm}$ for PD-L1 negatives, $P=0.0002)$ and was linearly associated with increasing depth of invasion $(P=0.0003)$. PD-L1-positive cases were more likely to display CD8+ lymphocytes (60 vs $28 \% P=0.0047$ ). The presence of CD8+ lymphocytes correlated significantly with depth of invasion $>1 \mathrm{~mm}(P=0.022)$. On multivariate analysis, PD-L1 was $6.14 \times$ more likely to be expressed in mixed DM than pure DM $(P=0.0131), C D 8+$ staining was $6.22 \times$ more likely in PDL1 positive cases than in PD-L1 negative $(P=0.0118)$, and tumor depth was associated with greater odds of PDL1 expression $(O R=1.61, P=0.0181)$. PD-L2 expression was observed in $48 \%$ of cases but did not correlate with any variables. Correlation of tumoral PD-L1 with increased depth and CD8+ lymphocytes implicates the tumoral immune microenvironment with advancing disease in DM. Enhanced tumoral PD-L1 expression in the mixed cytomorphological variant provides an insight into the differential pathogenesis of the subtypes and suggests that these patients are likely better candidates for anti-PD/PD-L1 therapy.

Modern Pathology (2017) 30, 357-369; doi:10.1038/modpathol.2016.210; published online 13 January 2017

Correspondence: Dr M Mahalingam MD, PhD, FRCPath, Dermatopathology Section, Department of Pathology and Laboratory Medicine (113), VA Integrated Service Networks (VISN1), 1400 VFW Parkway, West Roxbury, MA, 02132, USA.

E-mail: me@meeramahalingam.com

Received 15 September 2016; revised 16 November 2016; accepted 18 November 2016; published online 13 January 2017
Desmoplastic melanoma (DM), an uncommon morphological variant of melanoma, is associated with old age, chronic sun exposure, and location on the head and neck. ${ }^{1,2}$ Although relatively rare, the incidence of DM appears to be on the rise with a $4.6 \%$ annual increase noted from 1992 to $2007 .^{2}$ 
Overall, the prognosis of DM is distinct from that of non- DM s and is impacted by its histopathological classification into either the pure or mixed subtypes. ${ }^{3}$ If $>90 \%$ of the tumor is composed of cells with a spindled cytomorphology, then it is considered pure DM, and if the spindled cytomorphology is $<90 \%$ with the rest composed of tumoral cells with an epithelioid cytomorphology, it is considered mixed DM. ${ }^{3,4}$ This distinction is of clinical relevance as it is now understood that the mixed DM subtype is more prone to undergo metastasis than pure DM and is associated with increased mortality. ${ }^{3,5}$ In addition to prognostic differences between mixed DM and pure DM, underlying differences in the tumor biology exist that may, in part, explain the different clinical behaviors of the subtypes. ${ }^{6-10}$ Notably, pure DM appears to lack BRAF-V600E and other common driver mutations while mixed $\mathrm{DM}$ can be $B R A F$ mutated, albeit at a reduced rate compared with more common melanoma variants. ${ }^{6,10}$ These differences present important considerations in the therapeutic management of advanced cases of DM.

The lower incidence of $B R A F$ mutations in both mixed DM and pure DM compared with other melanoma variants suggests that they may be less amenable to available targeted therapies. Currently, immunomodulatory therapies that involve disrupting the programed death ligand-1 (PD-L1, CD274, B7H1) and programmed death receptor (PD1, CD273, B7-DC) axis present a promising alternative to targeted therapy in non-BRAF mutated melanomas. Under non-pathological conditions, the surface glycoprotein PD-L1 is expressed by antigenpresenting cells (APCs) and serves as an immune checkpoint' providing inhibitory input to cytotoxic T lymphocytes (CD8) and other immune effector cells via the PD1 receptor. The resultant reduction in CD8+ lymphocyte proliferation serves to promote self-tolerance. ${ }^{11}$ Tumors that express PD-L1 are able to use this mechanism to evade an antitumor immune response. Additionally, in some cancers tumor-infiltrating monocytes may be the primary source of inhibitory PD-L1 signaling. ${ }^{12}$ The disruption of PD1/PD-L1 signaling by therapeutic monoclonal antibodies (Nivolumab and Pembrolizumab) has resulted in durable responses and significant improvements in overall and progression-free survival in advanced melanoma, underscoring the clinical importance of this pathway. ${ }^{13-15}$ Attempts to discover predictive markers of response to anti-PD1/PDL1 therapy have revealed the importance of preexisting CD8+ tumor-infiltrating lymphocytes (tumorinfiltrating lymphocytes) and tumoral PD-L1 expression in this regard. ${ }^{16-18}$ Notably, Taube et al ${ }^{18}$ reported that tumoral PD-L1 expression was expressed in $53 \%$ of melanomas biopsied (type not specified) and that positive tumoral PD-L1 expression (membranous expression in $\geq 5 \%$ of melanoma cells) correlated significantly with clinical benefit to anti-PD1/PD-L1 therapy. These findings highlight the important role the use of immunohistochemistry to evaluate PD-L1 expression and CD8+ tumor-infiltrating lymphocyte status is likely to have in selecting candidates for PD1/L1 blockade.

Recently, in a retrospective analysis of patients treated with anti-PD1/PD-L1 therapies it was shown that patients with metastatic DM have higher response rates than metastases from other variants of melanoma. ${ }^{19}$ However, information regarding patterns of pretreatment PD-L1 expression in DM and its relationship to the pure DM and mixed DM subtypes does not, to date, exist. Given this, we sought to evaluate patterns of both tumoral PD-L1 and PD-L2 (a homologous surface glycoprotein to PD-L1 normally limited to macrophages and dendritic cells) in a cohort of primary DM samples. Additionally, we sought to correlate tumoral PD-L1/L2 expression to CD8+ tumor-infiltrating lymphocyte status as well as established histopathological prognosticators. Finally, select genetic parameters: an activating polymorphism in the glial cell line-derived neurotrophic factor (GDNF) tyrosine receptor kinase (RET) at codon G691S (RETp) and telomerase reverse transcripter (TERT) mutations, which have been implicated as genetic drivers in $\mathrm{DM}^{9,10}$ were evaluated for potential correlations with tumoral PD-L1/L2 expression.

\section{Materials and methods}

In this IRB-approved study, archival annotated (BRAF, RETp, and TERT) tissue samples with a diagnosis of primary DM were evaluated and 86 cases identified as fulfilling the criteria for inclusion (sufficient tissue for immunohistochemical analyses). Cases were subclassified as mixed DM or pure DM based on the criteria established by George et al. ${ }^{5}$ Briefly, cases in which $>90 \%$ of the tumor displayed a desmoplastic morphology were categorized as pure DM while cases in which $10-90 \%$ had a conventional morphology were classified mixed DM. All patient data were de-identified. Histological sections of all samples were reviewed by two board-certified dermatopathologists (initial sign-out on all was performed by a dermatopathologist and all cases re-reviewed and the diagnosis confirmed by the senior author, MM).

\section{Immunohistochemical Analysis}

Formalin-fixed, paraffin-embedded tissue from primary DM s $(n=86)$ were baked at $65^{\circ} \mathrm{C}$ for $30 \mathrm{~min}$. Antibodies used were as follows: PD-L1, clone SP263, obtained predilute from Ventana (Ventana Medical Systems, Tuscon, AZ, USA); PD-L2, clone no. 176611 (R\&D Systems, Minneapolis, MN, USA) used at a dilution of 1:2000; and CD8, clone SP57 obtained predilute from Ventana Medical Systems (Ventana). For PD-L1, sections were immunostained 

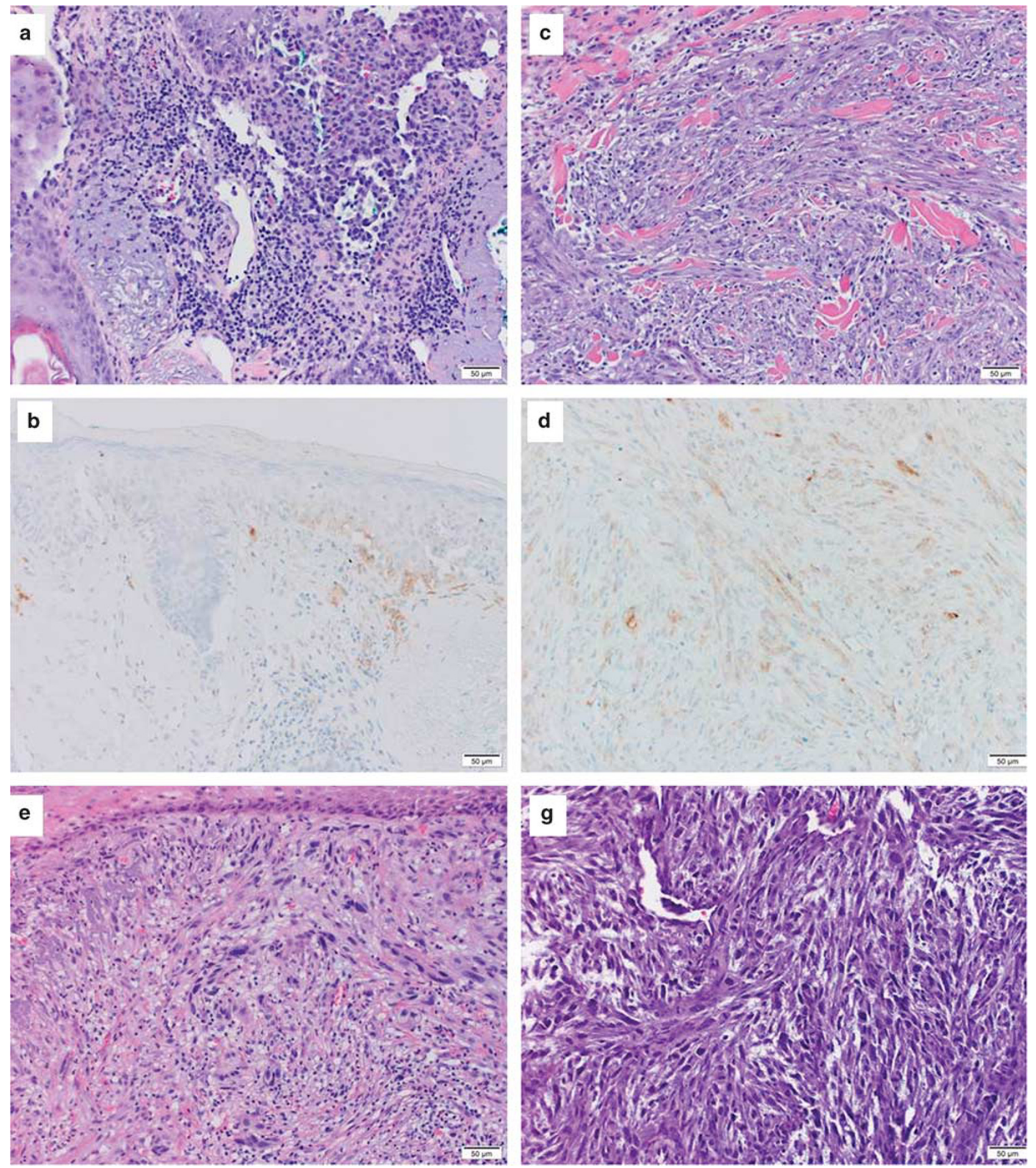

Figure 1 Representative examples of mixed desmoplastic melanoma cases from study with varied PD-L1 immunohistochemical scores. (a, b) Case 3: (a) H\&E; (b) PD-L1 (raw score 1, 5\%). (c, d) Case 58: (c) H\&E; (d) PD-L1 (raw score 2, > 5-20\%). (e, f) Case 55: (e) H\&E (f); PDL1 (raw score 3, > 20-50\%); (g, h) Case 76: (g) H\&E; (h) PD-L1, (raw score 4, >50\%).

using Ventana Optiview, while for PD-L2 and CD8, immunostaining was performed using Ventana Ultraview, UV-DAB. All immunostained slides were reviewed by and scored by the senior author in a blinded manner.
For both PD-L1 and PD-L2, positive scoring was determined using previously established cutoffs that have been suggested to predict response to PD1/PDL1 blockade ${ }^{18,20}$ Briefly, percentages of tumor cells displaying membranous staining were quantified as 

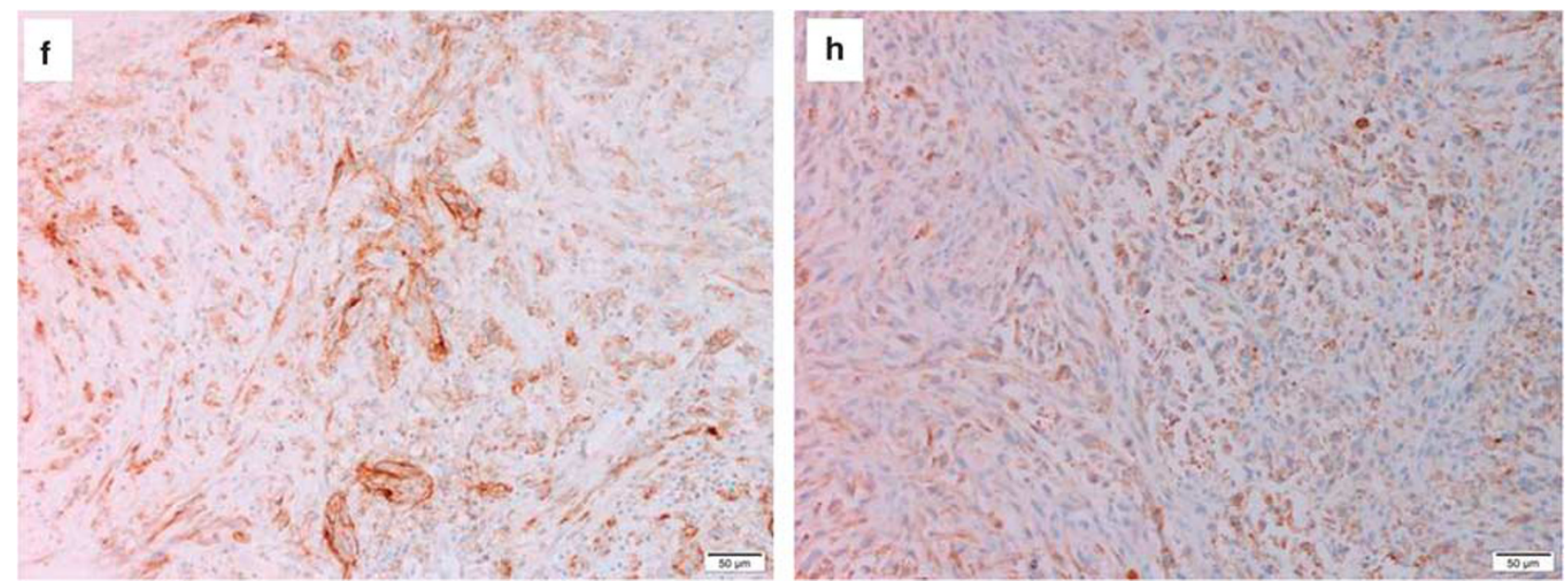

Figure 1 Continued

either $<5 \%, 5 \%,>5-20 \%,>20-50 \%$, or $>50 \%$ of the tumor bulk and then each case was assigned a score of $0-4$, respectively. Cases with a score $\geq 1$ were considered positive. CD8+ lymphocyte status was determined by semiquantitative scoring of stained immune infiltrates. Specifically, a score of $0-3$ was assigned to each case, where $0=$ no immune infiltrate present, $1=$ perivascular infiltration only, $2=$ perivascular + peritumoral and/or interstitial infiltration, and $3=$ diffuse, tumor obscuring infiltration. Scores of 2 or 3 were considered positive for CD8+ lymphocyte status. Formalin-fixed paraffinembedded tissue blocks of human placenta (PD-L1) and tonsil (PD-L2 and CD8) were used as positive controls.

\section{Statistical Analysis}

Differences in cohort demographic, histopathological variables, and genetic mutations were stratified by PD-L1, PD-L2, and CD8+ tumor-infiltrating lymphocyte status. To ascertain the relationship between each of the two PD ligands, CD8+ tumor-infiltrating lymphocyte status and desmoplastic subtype, a univariate analysis was used to identify potential confounders where cohort characteristics differed by both the PD-L1/2 expression and CD8+ tumorinfiltrating lymphocyte status. $P$-values were generated using Pearson's chi-square where expected frequencies exceeded 5, otherwise a Fisher's exact was used. The associations between DM thickness and expression of PD-L1 and PD-L2 were separately compared using an exact Wilcoxon to compare median thickness. A Mantel-Haenszel chi-square was used to test for linear trend by tumor depth.

Multivariable logistic regression was used to control for any potential confounders of PD-L1/2 expression, DM subtype, CD8+ tumor-infiltrating lymphocyte status, and other histopathological variables. A likelihood ratio test was used to check the overall fitness of the multivariable logistic model. Confounding was considered to be present if two mutually associated factors were also both correlated with the outcome of interest or where inclusion of both factors in a multivariable logistic model changed the odds ratio (percent confounding) by $>10 \%$. All statistical analyses were two-sided at alpha of 0.05 and carried out in SAS (SAS/STAT User's Guide, Version 9.4; SAS Institute, Cary, NC, USA).

\section{Results}

\section{Clinical and Histopathological Data}

Of the 86 cases of primary DM s studied, 58 were male and 28 were female. The median age at the time of diagnoses was 71 (interquartile range $=68$ ) years. Regarding anatomical location, 48 (55\%) came from the head and neck while 38 (45\%) were from nonhead-and-neck locations. Overall, 50 cases were classified as pure DM (58\%) while 36 cases were classified as mixed DM (42\%). Per previously established criteria, cases of mixed DM were further subclassified into mixed DM-DM predominant with the DM component being at least $50 \%$ but $<90 \%$ and mixed DM-conventional melanoma predominant, with the DM component constituting $>10 \%$ but $<50 \% .{ }^{5}$ Using these cutoffs, 14 of the 36 mixed DM cases were of the mixed DM-desmoplastic predominant subtype and 22 were of the mixed DM-conventional melanoma predominant subtype. In total, 74 cases $(86 \%)$ presented with a maximum depth of invasion $>1 \mathrm{~mm}$ with the most frequent AJCC thickness observed being $2-4 \mathrm{~mm} \quad(38 \%$ of cases). A junctional component was absent in 31 $(36 \%)$, ulceration was present in $7(8 \%)$, mitotic figures were identified in 54 (63\%), host response 

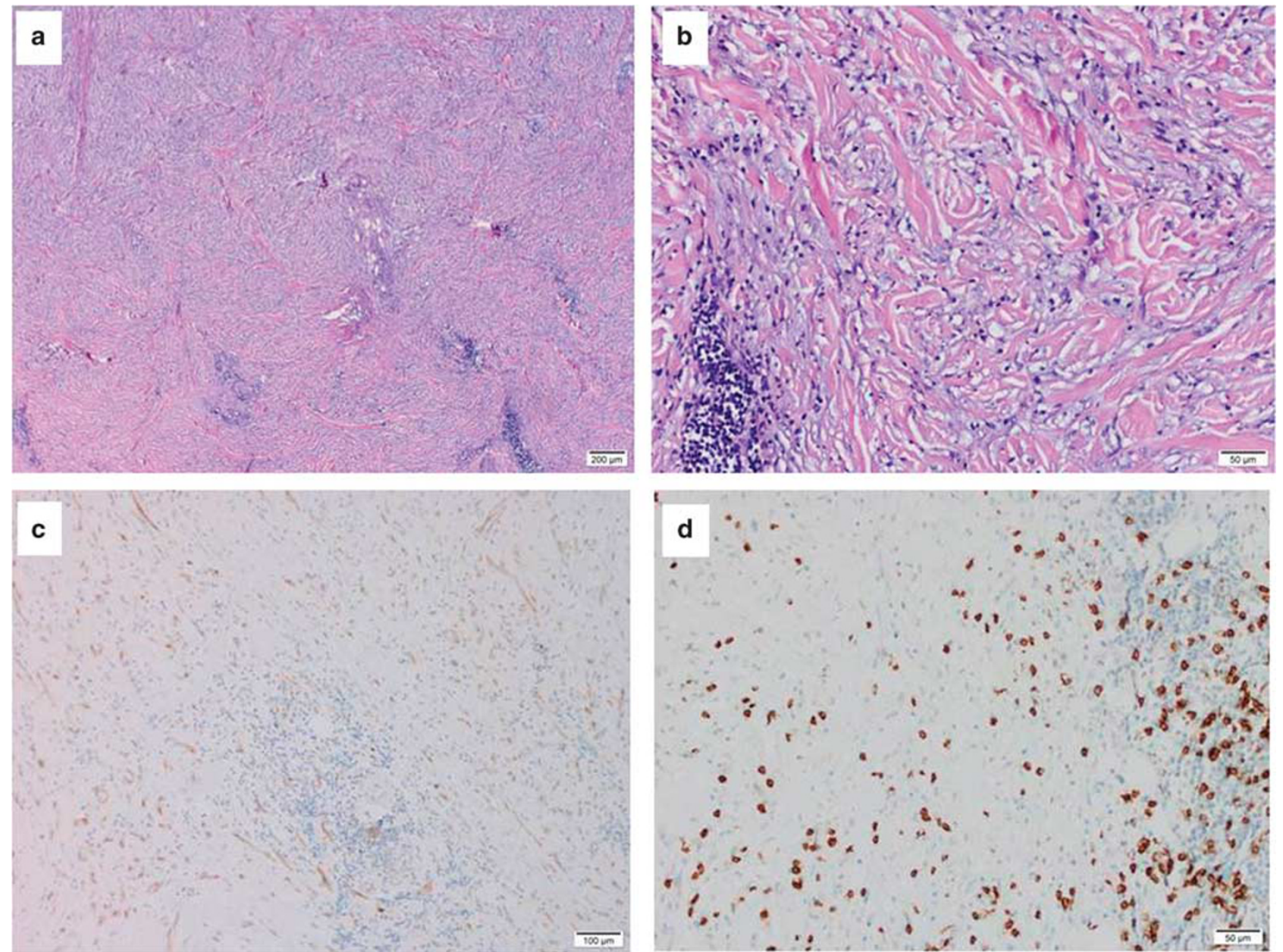

Figure 2 Representative example of pure desmoplastic melanoma case from study (a-d), Case 59. (a, b) H\&E $\times 4 / \times 20$. (c) Immunohistochemical stain PD-L1 (raw score 3). (d) Immunohistochemical stain CD8 (raw score 3).

was identified in $32(37 \%)$, and perineural invasion was observed in 49 (57\%) of cases. Of genetic variables previously annotated, 4 cases (5\%) exhibited mutations in BRAF, 21 exhibited polymorphisms in RET (RETp, 28\%), and 25 exhibited TERT promoter mutations $(35 \%)$.

\section{PD-L1 Expression}

The overall distribution with regards to tumoral PD-L1 expression was as follows: 43 (51\%) displayed $<5 \%$ expression, $20(24 \%)$ displayed $5 \%$ expression, $2(2 \%)$ displayed $5-20 \%$ expression, 7 (8\%) displayed $>20-50 \%$ expression, and 13 (15\%) displayed $>50 \%$ expression (representative examples in Figures 1 and 2). Using previously established cutoffs that have been suggested to predict response to PD1/PD-L1 blockade, 42 of the 85 cases (49\%, expression data unavailable from 1 case) were scored positive for PD-L1 expression.

On univariate analysis, PD-L1 expression was associated with a greater depth of invasion as measured by multiple criteria (Table 1). Notably, of all cases that expressed PD-L1, only 1 (2\%) had a greatest depth of $\leq 1 \mathrm{~mm}$, compared with $11(25 \%)$ of PD-L1-negative cases $(P=0.0034)$. When evaluated by AJCC guidelines, PD-L1 showed a significant linear association with increasing depth $(P=0.0028$; linear trend, $P=0.0003$ ). Additionally, the median depths of DMs with tumoral PD-L1 expression were significantly greater than those with absent PD-L1 expression (median $=3.29$, interquartile range $=1.75$ vs median $=1.75$, interquartile range $=2.04$; $P=0.0002)$. PD-L1 expression was associated with perineural invasion, with 31 (65\%) of PD-L1-positive cases displaying perineural invasion compared with $17(35 \%)$ of PD-L1-negative cases $(P=0.0014)$. In terms of cytomorphology, mixed DM demonstrated 1.5 times the rate of PD-L1 expression compared with pure DM (61 vs $41 \%$; $P=0.06$ ) before controlling for potential confounders. Although not achieving statistical significance, host response $59 \%$ in PD-L1 positive vs $41 \%$ in PD-L1 negative cases; $P=0.1534)$ and TERT mutational status $(67 \%$ mutated in PD-L1 positive vs 33\% in PD-L1 negative cases; $P=0.1827$ ) were found to be marginally 
Table 1 PD-L1/L2 expression and histopathological variables in desmoplastic melanoma

\begin{tabular}{|c|c|c|c|c|c|c|}
\hline Variable & $\begin{array}{c}P D-L 1^{\mathrm{a}} \text { positive, No. (\%), } \\
\mathrm{n}=42\end{array}$ & $\begin{array}{c}\text { PD-L1 negative, No. (\%) } \\
\mathrm{n}=43\end{array}$ & $\mathrm{P}$-values ${ }^{\mathrm{b}}$ & $\begin{array}{c}P D-L 2{ }^{\mathrm{C}} \text { positive, No. } \\
\mathrm{n}=41\end{array}$ & $\begin{array}{c}\text { PD-L2 negative, No. (\%), } \\
\mathrm{n}=38\end{array}$ & P-values \\
\hline \multicolumn{7}{|l|}{ Gender } \\
\hline Male, $n=58$ & $30(51.7)$ & $28(48.3)$ & \multirow[t]{2}{*}{0.5320} & $30(53.6)$ & $26(46.4)$ & \multirow{2}{*}{0.6424} \\
\hline Female, $n=28$ & $12(44.4)$ & $15(55.6)$ & & $11(47.8)$ & $12(52.2)$ & \\
\hline \multicolumn{7}{|l|}{ Anatomical site } \\
\hline Head/neck, $n=48$ & $24(50.0)$ & $24(50.0)$ & \multirow[t]{2}{*}{0.9017} & $27(58.7)$ & $19(41.3)$ & \multirow[t]{2}{*}{0.1534} \\
\hline Other, $n=38$ & $18(48.7)$ & $19(51.3)$ & & $14(42.4)$ & $19(57.6)$ & \\
\hline \multicolumn{7}{|l|}{ Desmoplastic variant } \\
\hline Pure, $n=50$ & $20(40.8)$ & $29(59.2)$ & \multirow[t]{2}{*}{0.0644} & $22(48.9)$ & $23(51.1)$ & \multirow[t]{2}{*}{0.5379} \\
\hline Mixed, $n=36$ & $22(61.1)$ & $14(38.9)$ & & $19(55.9)$ & $15(44.1)$ & \\
\hline $\begin{array}{l}\text { Median greatest } \\
\text { thickness }(\mathrm{mm})\end{array}$ & 3.29, interquartile range $=3.58$ & 1.75 , interquartile range $=2.04$ & 0.0002 & 2.44 , interquartile range $=2.00$ & 2.27, interquartile range $=2.77$ & 0.5479 \\
\hline \multicolumn{7}{|l|}{ AJCC thickness } \\
\hline$<1 \mathrm{~mm}, n=12$ & $1(8.3)$ & $11(91.7)$ & \multirow[t]{4}{*}{0.0028} & $6(54.6)$ & $5(45.5)$ & \multirow[t]{4}{*}{0.6493} \\
\hline $1-2 \mathrm{~mm}, n=20$ & $8(40.0)$ & $12(60.0)$ & & $8(40.0)$ & $12(60.0)$ & \\
\hline $2-4 \mathrm{~mm}, n=33$ & $18(56.3)$ & $14(43.7)$ & & $18(58.1)$ & $13(41.9)$ & \\
\hline$>4 \mathrm{~mm}, n=21$ & $15(71.4)$ & $6(28.5)$ & & $9(52.9)$ & $8(47.1)$ & \\
\hline \multicolumn{7}{|l|}{ Greatest thickness } \\
\hline$>1 \mathrm{~mm}, n=74$ & $41(56.2) 1$ & $32(43.8)$ & \multirow[t]{2}{*}{0.0034} & $35(51.5)$ & $33(48.5)$ & \multirow{2}{*}{0.8498} \\
\hline$<1 \mathrm{~mm}, n=12$ & $(8.3)$ & $11(91.7)$ & & $6(54.6)$ & $5(45.4)$ & \\
\hline \multicolumn{7}{|l|}{ Mitosis } \\
\hline Present, $n=54$ & $28(52.8)$ & $25(47.2)$ & \multirow[t]{2}{*}{0.4172} & $27(57.5)$ & $20(42.6)$ & \multirow[t]{2}{*}{0.2317} \\
\hline Absent, $n=32$ & $14(43.8)$ & $18(56.2)$ & & $14(43.8)$ & $18(56.2)$ & \\
\hline \multicolumn{7}{|l|}{ Ulceration } \\
\hline Present, $n=7$ & $5(71.4)$ & $2(28.6)$ & \multirow[t]{2}{*}{0.2652} & $6(85.7)$ & $1(14.3)$ & \multirow[t]{2}{*}{0.0667} \\
\hline Absent, $n=79$ & $37(47.4)$ & $41(52.6)$ & & $35(48.6)$ & $37(51.4)$ & \\
\hline \multicolumn{7}{|l|}{ Host response } \\
\hline Present, $n=32$ & 19 (59.4) & $13(40.6)$ & \multirow[t]{2}{*}{0.1534} & $18(60.0)$ & $12(40.0)$ & \multirow[t]{2}{*}{0.2595} \\
\hline Absent, $n=54$ & $23(43.3)$ & $30(56.7)$ & & $23(46.9)$ & $26(53.1)$ & \\
\hline \multicolumn{7}{|l|}{ Perineural invasion } \\
\hline Present, $n=49$ & $31(64.6)$ & $17(35.4)$ & \multirow[t]{2}{*}{0.0014} & $26(59.1)$ & $18(40.9)$ & \multirow[t]{2}{*}{0.1514} \\
\hline Absent, $n=37$ & $11(29.7)$ & $26(70.3)$ & & $15(42.9)$ & $20(57.1)$ & \\
\hline Junctional componer & & & & & & \\
\hline Present, $n=55$ & $25(46.3)$ & $29(53.7)$ & 0.4483 & $27(51.9)$ & $25(48.1)$ & 0.9952 \\
\hline Absent, $n=31$ & $17(54.8)$ & $14(45.2)$ & & $14(43.8)$ & $13(48.2)$ & \\
\hline CD8+ tumor-infiltrat & lymphocyte status ${ }^{\mathrm{d}}$ & & & & & \\
\hline Positive, $n=55$ & $33(60.0)$ & $22(40.0)$ & 0.0047 & $26(48.2)$ & $28(51.9)$ & 0.3268 \\
\hline Negative, $n=30$ & $8(27.6)$ & $21(72.4)$ & & $15(60.0)$ & $10(40.0)$ & \\
\hline
\end{tabular}




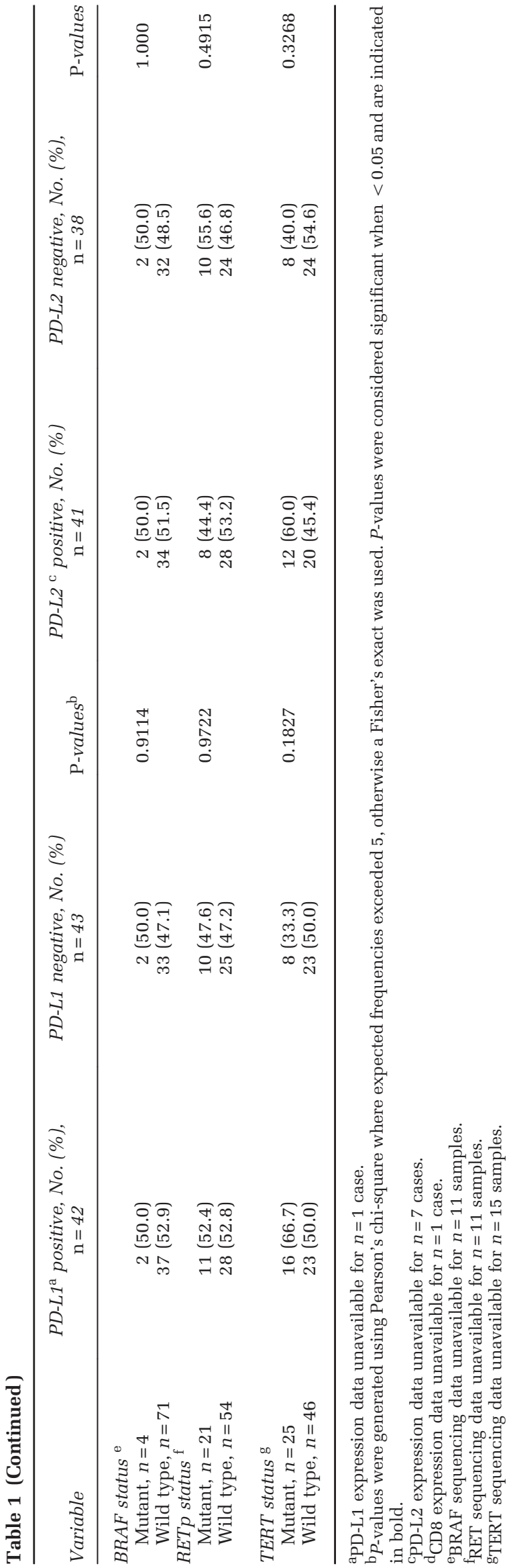

associated with PD-L1 expression and were therefore included in multivariate analysis. Though PD-L1 expression was not associated with host response on H\&E, immunohistochemical staining for CD8+ tumor-infiltrating lymphocyte status revealed a significant correlation, as cases scoring positive for CD8+ lymphocytes were two times more likely to express PD-L1 than those lacking CD8+ tumorinfiltrating lymphocytes (60 vs 28\%, $P=0.0047$ ). Ulceration, mitoses, BRAF mutational status, and RETp mutational were not associated with PD-L1 expression. No correlation was found between both PD-L1 and PD-L2 expression levels.

Histopathological features, immunological effector cell presence, and mutational status were identified as potential confounders and controlled for in a multiple variable analysis of PD-L1 (Table 2). Overall, DM subtype, depth, perineural invasion, host response, CD8+ tumor-infiltrating lymphocyte expression, and TERT promoter mutation status predicted PD-L1 expression $\left(\chi^{2}=33.62, \mathrm{df}=6\right.$, $P<0.0001)$. DM thickness was included in the logistic regression as a continuous measurement owing to a paucity of PD-L1-positive samples $<1 \mathrm{~mm}$. When controlling for these potential cofounders, the mixed DM variant was 6.14 times as likely to express PD-L1 than the pure variant $(P=0.0131)$ and cases with a positive CD8+ lymphocyte status were 6.22 times as likely to express PD-L1 than those cases in which CD8+ staining was absent $(P=0.0118)$. A $1-\mathrm{mm}$ increase in tumor depth was associated with 1.61-fold greater odds of PD-L1 expression $(P=0.0181)$. The association between PD-L1 expression and perineural invasion was not significant after controlling for potential cofounders $(P=0.13)$.

\section{PD-L2 Expression}

Using the same cutoffs as described for PD-L1, expression of PD-L2 was observed in 41/79 (52\%, expression data unavailable for 7 cases) of samples. The overall distribution with regards to PD-L2 cutoffs was as follows: $38(48 \%)$ displayed $<5 \%$ expression, $24(30 \%)$ displayed 5\% expression, 10 $(13 \%)$ displayed $5-20 \%$ expression, 5 (6\%) displayed $>20-50 \%$ expression, and $2(3 \%)$ displayed $>50 \%$ expression.

Although not achieving statistical significance, on univariate analysis PD-L2 was marginally associated with ulceration, and its expression was observed in $86 \%$ of ulcerated cases vs $49 \%$ of non-ulcerated $(P=0.0667)$ (Table 1). PD-L2 was not significantly associated with any of the histopathological variables or genetic variables analyzed. When controlling for potentially confounding variables in a multivariable regression model, PD-L2 remained unassociated with ulceration $(\mathrm{OR}=6.81$, $P=0.0874)$. 
Table 2 Multivariable analysis of PD1 and mixed desmoplastic variant association controlling for histopathological, genetic, and immunological confounders

\begin{tabular}{|c|c|c|c|c|}
\hline Parameter $^{\mathrm{a}}$ & S.e. & Odds ratio ${ }^{\mathrm{b}}$ & $95 \%$ confidence limits & $\mathrm{P}$-values ${ }^{\mathrm{C}}$ \\
\hline Mixed desmoplastic variant & 0.3660 & 6.14 & $1.46-25.80$ & 0.0131 \\
\hline Tumor depth (mm) & 0.2003 & 1.61 & $1.08-2.38$ & 0.0181 \\
\hline Perineural invasion & 0.3550 & 2.97 & $0.74-6.64$ & 0.1250 \\
\hline Host response & 0.3467 & 1.71 & $0.44-6.64$ & 0.4406 \\
\hline Positive CD8+ tumor-infiltrating lymphocyte status & 0.3629 & 6.22 & $1.50-25.79$ & 0.0118 \\
\hline TERT promoter mutation & 0.3469 & 1.28 & $0.33-4.98$ & 0.7225 \\
\hline
\end{tabular}

${ }^{\mathrm{a}}$ Logistic regression on $n=69$ samples.

${ }^{\mathrm{b}}$ Odds ratio indicates association between PD-L1 expression and a given parameter.

${ }^{\mathrm{C}} P$-values were considered significant when $<0.05$ and are indicated in bold.

\section{Cytotoxic CD8+ Tumor-Infiltrating Lymphocytes}

Overall, 55/85 (65\%) scored positive for CD8+ tumor-infiltrating lymphocyte status. The distribution of CD8+ tumor-infiltrating lymphocyte scoring was as follows: 9 (11\%) of cases scored 0 (did not display any CD8+ tumor-infiltrating lymphocytes), $21(24 \%)$ scored 1 (perivascular CD8+ tumorinfiltrating lymphocytes), 38 (45\%) scored 2 (perivascular+peritumoral and/or interstitial CD8+ tumor-infiltrating lymphocytes), and 17 (20\%) scored 3 (diffuse CD8+ tumor-infiltrating lymphocytes).

On univariate analysis, CD8+ tumor-infiltrating lymphocyte status was associated with a tumor thickness $>1 \mathrm{~mm}$ with $51(70 \%)$ of DMs with a maximum depth $>1 \mathrm{~mm}$ having positive CD8+ tumor-infiltrating lymphocyte status vs 4 (33\%) of DMs with a maximum depth $\leq 1 \mathrm{~mm}(P=0.0217)$ (Table 3). Host response as seen on $\mathrm{H} \& \mathrm{E}$ was also associated with CD8+ tumor-infiltrating lymphocyte status as $26(81 \%)$ of DMs with an observed host response scored positive for CD8+ tumor-infiltrating lymphocyte vs 29 (53\%) of DMs without an observed host response $(P=0.0187)$. CD8+ tumor-infiltrating lymphocyte status was not associated with any other histopathological or genetic variable analyzed.

Although both tumor depth and host response were associated with CD8 tumor-infiltrating lymphocyte expression, there was no empirical evidence of confounding. DM tumors $>1 \mathrm{~mm}$ were no more likely than those $\leq 1 \mathrm{~mm}$ to have shown a host response (3/12 vs 29/74; $P=0.5221)$. Furthermore, a subanalysis, comparing a multiple logistic to simple logistic regression, indicated that neither was confounding the other clinicopathological factor's association with CD8+ tumor-infiltrating lymphocyte by $>10 \%$.

\section{Discussion}

It is increasingly understood that DM presents with a distinct set of underlying genetic and phenotypic abnormalities when compared with conventional

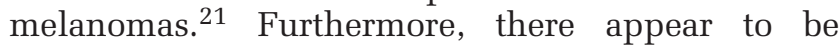

differences in the pattern of driver mutations seen between the pure and mixed DM variants. ${ }^{6,9,10}$ These differences have important therapeutic and prognostic implications. Notably, the well-documented reduced rate of $B R A F$ mutations in DM (only 4/75 cases had $B R A F$ mutations in the present study) compared with other melanoma variants indicates that patients with DM are poor candidates for BRAFtargeted therapies. ${ }^{10,21}$ However, the recent work by Shain et $a l^{21}$ has shown in a study of 42 cases of DM that the average overall mutational burden is one of the highest observed in any cancer type (62 mutations/Mb). Given that successful response to antiCTLA4 immune-checkpoint blockade in melanoma has been shown to be associated with the expression of neo-antigens, the products of mutated genes displayed by tumor cells on MHC class 1 surface proteins, some have hypothesized that the increased mutational burden in DM will be associated with an enhanced response to anti-PD1/PD-L1 therapy. ${ }^{22-24}$ In keeping with this, one report from a cohort of 1000 metastatic melanoma patients treated with anti-PD1/ PD-L1 antibodies has shown that patients with metastatic DM had a better response rate compared with the overall cohort. ${ }^{19}$ Further supporting a role for mutational burden in predicting response to antiPD1/PD-L1 therapy is the recent findings by Madore et $a l^{25}$ that, in the setting of stage III melanoma lymph node biopsies, PD-L1-negative status is associated with a lower mutational burden.

In an effort to further elucidate the therapeutically relevant phenotypic characteristics that define DM, as well the functional differences between the pure and mixed variant, we sought to evaluate patterns of tumoral PD-L1/PD-L2 expression and CD8+ tumorinfiltrating lymphocyte status in a cohort of DMs. We found that $42 / 85(49 \%)$ of the DMs studied expressed tumoral PD-L1 in $\geq 5 \%$ of cells. A recent study by Kakavand et $a l^{26}$ in 60 patients with metastatic melanoma (2 of which were DM) found that $43 \%$ of the cases studied expressed tumoral PDL1 using a cutoff of $\geq 1 \%$ tumoral expression for positivity. This is similar to an earlier study by Madore et $a l^{27}$ also using the $\geq 1 \%$ tumoral PD-L1 expression cutoffs which found that $51 \%$ of the 43 
primary melanomas (type unspecified) studied were PD-L1 positive. Rodić et al ${ }^{20}$ found similar rates of PD-L1 expression, with $43 \%$ of 23 primary melano- mas studied (type unspecified) using the same $\geq 5 \%$ tumoral PD-L1 expression cutoffs as the present study. Our results, when taken in context with

Table 3 CD8+ lymphocyte status and histopathological variables in desmoplastic melanoma

\begin{tabular}{|c|c|c|c|}
\hline Variable & $\begin{array}{c}\text { CD8+ tumor-infiltrating lymphocyte positive }{ }^{\mathrm{a}} \text {, } \\
\text { No. (\%), } \mathrm{n}=55\end{array}$ & $\begin{array}{c}\text { CD8+ tumor-infiltrating lymphocyte negative, } \\
\text { No. }(\%), \mathrm{n}=30\end{array}$ & $\mathrm{P}$-values ${ }^{\mathrm{b}}$ \\
\hline \multicolumn{4}{|l|}{ Gender } \\
\hline Male, $n=58$ & $40(69.0)$ & $18(31.0)$ & \multirow[t]{2}{*}{0.2284} \\
\hline Female, $n=28$ & $15(55.6)$ & $12(44.4)$ & \\
\hline \multicolumn{4}{|l|}{ Anatomical site } \\
\hline Head/neck, $n=48$ & $30(62.5)$ & $18(37.5)$ & \multirow[t]{2}{*}{0.6279} \\
\hline Other, $n=38$ & $25(67.6)$ & $12(32.4)$ & \\
\hline \multicolumn{4}{|l|}{ Desmoplastic variant } \\
\hline Pure, $n=50$ & $30(61.2)$ & $19(38.8)$ & \multirow{2}{*}{0.4333} \\
\hline Mixed, $n=36$ & $25(69.4)$ & $11(30.6)$ & \\
\hline $\begin{array}{l}\text { Median greatest thickness } \\
(\mathrm{mm})\end{array}$ & 2.50 , interquartile range $=2.42$ & 2.25 , interquartile range $=3.03$ & 0.3279 \\
\hline \multicolumn{4}{|l|}{ AJCC thickness } \\
\hline$\leq 1 \mathrm{~mm}, n=12$ & $4(33.3)$ & $8(66.7)$ & \multirow{4}{*}{0.0723} \\
\hline $1-2 \mathrm{~mm}, n=20$ & $16(80.0)$ & $4(20.0)$ & \\
\hline $2-4 \mathrm{~mm}, n=33$ & $22(66.7)$ & $11(33.3)$ & \\
\hline$>4 \mathrm{~mm}, \mathrm{n}=21$ & $13(65.0)$ & $7(35.0)$ & \\
\hline \multicolumn{4}{|l|}{ Greatest thickness } \\
\hline$>1 \mathrm{~mm}, n=74$ & $51(69.9)$ & $22(30.1)$ & \multirow[t]{2}{*}{0.0217} \\
\hline$\leq 1 \mathrm{~mm}, n=12$ & $4(33.3)$ & $8(66.7)$ & \\
\hline \multicolumn{4}{|l|}{ Mitosis } \\
\hline Present, $n=54$ & $35(66.0)$ & $18(34.0)$ & \multirow[t]{2}{*}{0.7409} \\
\hline Absent, $n=32$ & $20(62.5)$ & $12(37.5)$ & \\
\hline \multicolumn{4}{|l|}{ Ulceration } \\
\hline Present, $n=7$ & $5(71.4)$ & $2(28.6)$ & \multirow[t]{2}{*}{0.5239} \\
\hline Absent, $n=79$ & $50(64.1)$ & $28(35.9)$ & \\
\hline \multicolumn{4}{|l|}{ Host response } \\
\hline Present, $n=32$ & $26(81.3)$ & $6(18.8)$ & \multirow[t]{2}{*}{0.0187} \\
\hline Absent, $n=54$ & $29(53.7)$ & $24(44.4)$ & \\
\hline \multicolumn{4}{|l|}{ Perineural invasion } \\
\hline Present, $n=49$ & $31(64.6)$ & $17(35.4)$ & \multirow[t]{2}{*}{0.9785} \\
\hline Absent, $n=37$ & $24(64.9)$ & $13(35.1)$ & \\
\hline \multicolumn{4}{|l|}{ Junctional component } \\
\hline Present, $n=55$ & $36(65.5)$ & $19(34.5)$ & \multirow[t]{2}{*}{0.8449} \\
\hline Absent, $n=31$ & $19(63.3)$ & $11(36.7)$ & \\
\hline \multicolumn{4}{|l|}{ BRAF status ${ }^{\mathrm{C}}$} \\
\hline Mutant, $n=4$ & $4(100.0)$ & $0(0.0)$ & \multirow[t]{2}{*}{0.2900} \\
\hline Wild type, $n=71$ & $44(62.0)$ & $27(38.0)$ & \\
\hline \multicolumn{4}{|l|}{ RETp status ${ }^{\mathrm{d}}$} \\
\hline Mutant, $n=21$ & $13(61.9)$ & $8(38.1)$ & \multirow{2}{*}{0.8136} \\
\hline Wild type, $n=54$ & $35(64.81)$ & $19(35.2)$ & \\
\hline \multicolumn{4}{|l|}{ TERT status ${ }^{\mathrm{e}}$} \\
\hline Mutant, $n=25$ & $15(62.5)$ & $9(37.5)$ & \multirow[t]{2}{*}{0.8218} \\
\hline Wild type, $n=46$ & $30(65.2)$ & $16(34.8)$ & \\
\hline
\end{tabular}

${ }^{\mathrm{a}} \mathrm{CD} 8+$ expression data unavailable for $n=1$ case.

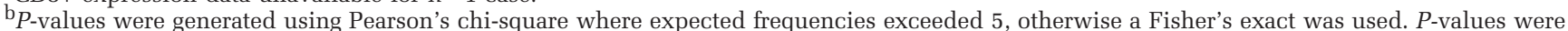
considered significant when $<0.05$ and are indicated in bold.

${ }^{\mathrm{C}}$ BRAFsequencing data unavailable for $n=11$ samples.

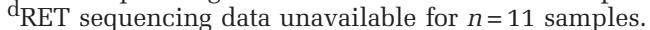

eTERT sequencing data unavailable for $n=15$ samples. 
previous observations, suggest that DM as a whole displays similar rates of PD-L1 expression to conventional melanomas.

In melanoma, the prognostic meaning of tumoral PD-L1 expression in the absence of immunotherapy is equivocal. In the setting of advanced stage melanoma, multiple studies have shown that tumors lacking PD-L1 expression have worse outcomes. ${ }^{28,29}$ However, others have shown that tumoral PD-L1 expression is a poor prognostic indicator. ${ }^{30-32}$ Therefore, it is difficult to draw a definitive conclusion about the prognostic value of PD-L1 expression in DM on its own. Even so, in the present study we observed significant correlations between tumoral PD-L1 expression and measurements of increasing tumor depth, an established prognosticator of more aggressive disease. Notably, there was a significant linear trend between increasing depth as established by AJCC guidelines and increasing rates of PD-L1 expression. These results could suggest that, in DM, PD-L1 expression may be associated with more aggressive disease. It has been shown that PD-L1 expression in melanomas may be an adaptive response to IFN- $\gamma$ secretion by CD8+ tumorinfiltrating lymphocytes. ${ }^{17,29}$ Given our finding that CD8+ tumor-infiltrating lymphocyte status was correlated with a maximum tumor depth $>1 \mathrm{~mm}$, an alternative explanation for the increased PD-L1 expression found in more invasive melanomas may be that it is an example of this adaptive response to IFN- $\gamma$ secretion by CD8+ tumor-infiltrating lymphocytes found more frequently in deeply invading tumors. This interpretation is repudiated by the finding that, in our multivariate analysis, the association between tumoral PD-L1 expression and maximum tumor depth $>1 \mathrm{~mm}$ was independently associated with PD-L1 expression even after controlling for CD8+ tumor-infiltrating lymphocyte status, which also remained significant. Aside from depth and CD8+ tumor-infiltrating lymphocyte status, perineural invasion was associated with tumoral PD-L1 expression (Table 1). However, this association was not significant after multivariate analysis. One possible explanation is that, as we have found previously, perineural invasion is associated with deeper DMs as these tumors are more likely to encounter nerve fibers at greater depths, hence the multivariate analysis controlled for the confounding influence of depth resulting in a loss of significance. ${ }^{7,33}$

The mixed and pure variants of DM differ not only in morphology but also in prevalence of select oncogenic mutations and in prognosis. ${ }^{5-10}$ The mixed variant is associated with both an increased rate of lymph node metastases and is enriched for known activating mutations in oncogenes. ${ }^{10}$ These differences suggest that the etiopathogenesis of pure DM and mixed DM may be distinct. After controlling for potential confounders in a multivariate analysis, we found that there was a greater than fivefold increase in the likelihood of PD-L1 expression in the mixed DM compared with pure DM. This association prompted us to re-evaluate cases of mixed DM to ascertain whether the pattern of PD-L1 staining was restricted to either the desmoplastic or conventional component of these tumors. We found that, of the mixed DMs that scored positive for PD-L1 expression, $17 / 22(77 \%)$ displayed staining confined to the conventional component and that all 22 of the PDL1-positive mixed DMs were of the conventional melanoma predominant subcategory of mixed DM (Figure 3). The confinement of PD-L1 expression to the conventional component in the mixed cytomorphological variant together with the increased frequency of PD-L1 positivity in the mixed $v s$ the pure subtype (as seen on multivariable analysis) underscores differences in adaptive immune resistance between the epithelioid and spindled melanoma phenotypes. The decreased propensity for lymph node metastasis in the pure subtype of DM suggests that PD-L1 expression may be a surrogate indicator of the more aggressive subtypes. Further underscoring the biological differences between desmoplastic subtypes is the genetic analyses by Jahn et $a 1^{10}$ showing that pure DM is more likely to display mutations in tumor-suppressor genes rather than oncogenes or to contain no known mutations, while the mixed subtype exhibited activating mutations in common conventional melanoma oncogenes (BRAF, NRAS, FGFR2, and ERBB2) albeit at a reduced rate. Our prior genetic analyses of DM has also revealed significant differences in the subtypes, with a higher proportion of cases of the mixed subtype exhibiting telomerase reverse transcripter promoter mutations. ${ }^{9}$ Our prior immunohistochemical analyses of DM has shown that the mixed subtype displays a significantly increased Ki-67 proliferative rate and increased expression of the oncogene KIT when compared with the pure subtype and that neurofibromin loss is more common in the pure subtype. ${ }^{6,8}$ Taken together with findings from the current study, these studies underscore that the DM subtypes have distinct genetic drivers.

Although the prognostic relevance of PD-L1 expression on its own may be equivocal, it is becoming clear that there is value in determining the presence of tumoral PD-L1 for predicting response to anti-PD1/PD-L1 immunotherapy. ${ }^{14,15,34}$ However, it should be noted that responses to antiPD1/PD-L1 therapy have been observed in some cases of PD-L1-negative melanoma of a variety of subtypes. ${ }^{35}$ Some have argued that, because CD8+ tumor-infiltrating lymphocytes are able to induce PD-L1 expression in tumor cells via IFN- $\gamma$ secretion, PD-L1 serves as a marker of an on-going CD8+ cytotoxic antitumor reaction and the subsequent adaptive immune resistance response in the tumor. ${ }^{17,29}$ The concept of adaptive immune resistance may be important in establishing predictors to anti-PD1/PD-L1 therapy as Temeh et $a l^{17}$ found that the presence of CD8+ tumor-infiltrating lymphocytes in conjunction with PD-L1 expression in 

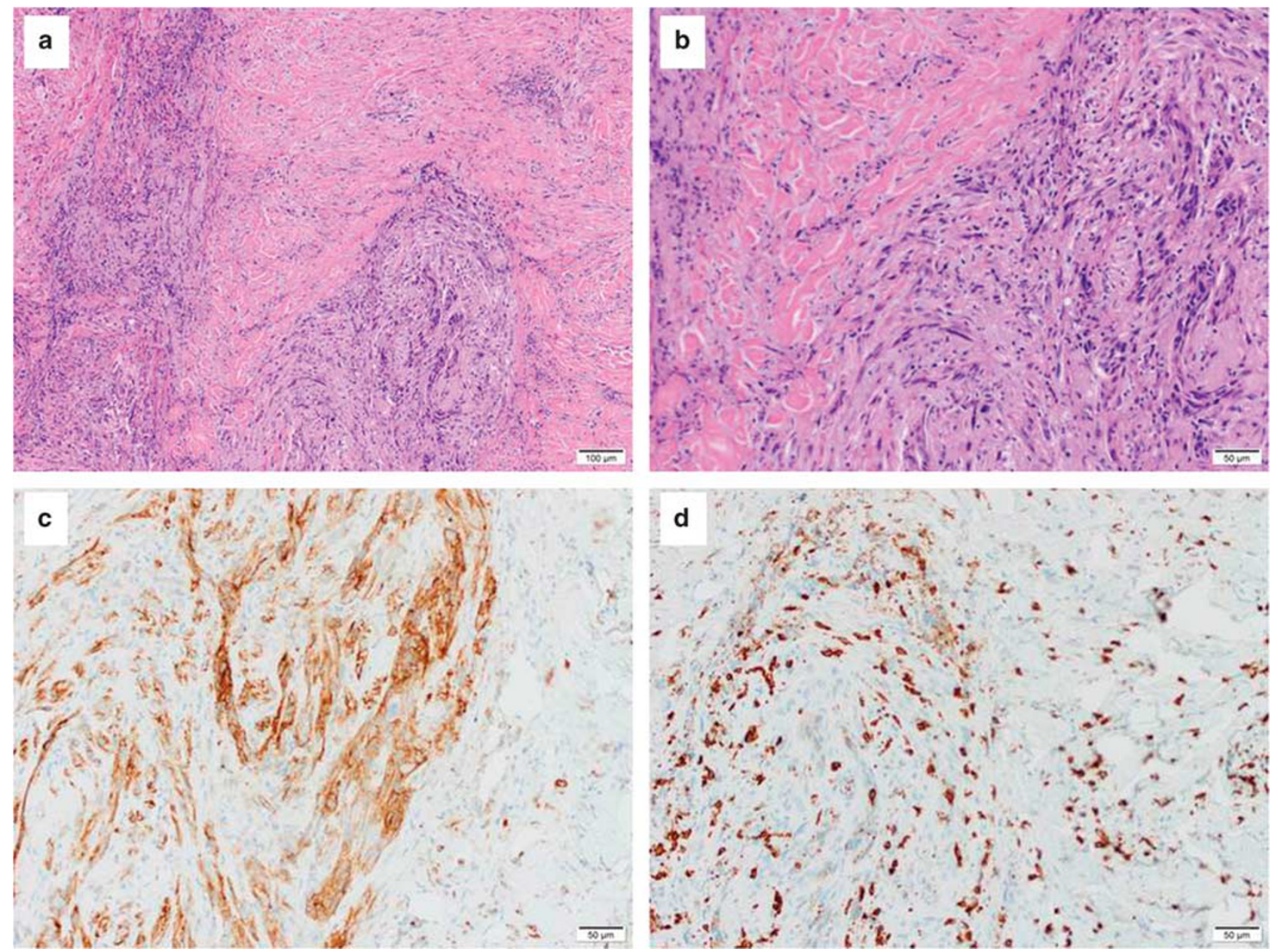

Figure 3 Representative example of mixed desmoplastic melanoma case with PD-L1 staining confined exclusively to conventional/ epithelioid component (a-d), Case 95. (a, b) H\&E $\times 10 / \times 20$. (c) Immunohistochemical stain PD-L1 (raw score 4). (d) Immunohistochemical stain CD8 (raw score 3).

pretreatment biopsies predicted response to antiPD1/PD-L1 therapy in metastatic melanomas of unspecified subtype. We found that, in both our univariate and multivariate analysis, PD-L1 expression is associated with a positive CD8+ tumorinfiltrating lymphocyte status in DM-indicative of an adaptive immune resistance phenomenon in DM.

PD-L2 is a homolog of PD-L1 and also acts as a ligand to PD1 that inhibits the activation of cytotoxic CD8+ tumor-infiltrating lymphocytes. ${ }^{36}$ Although we did observe tumoral PD-L2 expression in $52 \%$ of DMs studied, we did not find any significant correlation between PD-L2 and PD-L1 or with PDL2 and CD8+ tumor-infiltrating lymphocyte status. Tumoral PD-L2 expression did not correlate with any other histopathological or genetic variable studied. We did observe a marginal correlation between tumoral PD-L2 expression and ulceration $(P=0.0667)$. However, we are likely underpowered to detect the true effect of ulceration on PD-L2 expression as only 1/38 samples were both ulcerated and lacked PD-L2 expression. It is unlikely that the seven non-ulcerated cases that failed to stain for PD-L2 are differentially biasing the results. Therefore, this study may be an underestimate of the true association between ulceration and PD-L2 and future research is warranted. The role of PD-L2 in adaptive immune resistance and in predicting response to immune checkpoint blockade is currently unknown. Taube et $a 1^{18}$ found in a study of multiple cancer types, including melanomas (type unspecified), that PD-L2 expression correlated with PD-L1 status. However, we found no such correlation in DM, a finding that further underscores differences between $\mathrm{DM}$ and conventional melanoma.

Future investigations into the pathological and prognostic role of PD-L1/PD-L2 expression in DM may benefit from the use of patient-matched biopsies of both primary and metastatic lesions. As anti-PD1/ PD-L1 therapy is used in the setting of metastatic disease, PD-L1 positivity in biopsies from metastatic sites may be more predictive of response. Currently, many studies that have investigated the predictive value of PD-L1 expression for anti-PD1/PD-L1 
therapy have not differentiated between primary and metastatic biopsies. ${ }^{14,17}$ Taube et $a l^{29}$ have shown that there are similar rates of PD-L1 positivity in primary and metastatic biopsies (not patient matched) in the setting of conventional melanoma. Although a larger study by Madore et $a l^{27}$ found that in patient-matched melanoma biopsies PD-L1 expression was discordant between primary and metastatic lesions. Given the higher frequency of lymph node involvement in the mixed subtype together with findings from the current study, it is our contention that matched samples of the mixed cytomorphological variant are likely to demonstrate a similar PD-L1 profile. Although this observation requires further study and validation on larger sample sizes of mixed DM cases as well as matched samples, it may in part begin to explain the significant phenotypic differences between these two groups. Finally, although ours is the largest study on PD-L1 expression in primary DM, given the relative rarity of $\mathrm{DM}$, sample size remains a limitation. Nonetheless, given the marginally significant $(P=0.0644)$ association on univariate analysis of PDL1 expression with the mixed desmoplastic subtype, it is possible to hypothesize, from a scientific perspective alone, that such an association would achieve statistical significance with a larger cohort.

In conclusion, findings from the current study of a correlation of tumoral PD-L1 with increased depth of invasion, as well as the presence of CD8+ lymphocytes, implicate the tumoral immune microenvironment with advancing disease in DM. More importantly, our findings provide a biological explanation for the recent observation that patients with metastatic DM respond more favorably to anti-PD1/ PD-L1 therapy than other melanoma subtypes. Enhanced tumoral PD-L1 expression in mixed DM provides an insight into the differential pathogenesis of the subtypes and suggests that DM patients with this cytomorphological variant are likely better candidates for anti-PD/PD-L1 therapy.

\section{Disclosure/conflict of interest}

The authors declare no conflict of interest.

\section{References}

1 Chen LL, Jaimes N, Barker CA, et al. Desmoplastic melanoma: a review. J Am Acad Dermatol 2013;68: 825-833.

2 Feng Z, Wu X, Chen V, et al. Incidence and survival of desmoplastic melanoma in the United States, 19922007. J Cutan Pathol 2011;38:616-624.

3 Busam KJ, Mujumdar U, Hummer AJM, et al. Cutaneous desmoplastic melanoma: reappraisal of morphologic heterogeneity and prognostic factors. J Surg Pathol 2004;28:1518-1525.

4 Hawkins WG, Busam KJ, Ben-Porat L, et al. Desmoplastic melanoma: a pathologically and clinically distinct form of cutaneous melanoma. Ann Surg Oncol 2005;12:207-213.

5 George E, McClain SE, Slingluff CL, et al. Subclassification of desmoplastic melanoma: pure and mixed variants have significantly different capacities for lymph node metastasis. J Cutan Pathol 2009;36: 425-432.

6 Miller DD, Emley A, Yang S, et al. Mixed versus pure variants of desmoplastic melanoma: a genetic and immunohistochemical appraisal. Mod Pathol 2012;25: $505-515$.

7 Frydenlund N, Leone DA, Mitchell B, et al. Neurotrophin receptors and perineural invasion in desmoplastic melanoma. J Am Acad Dermatol 2015;72: 851-858.

8 Kadokura A, Frydenlund N, Leone DA, et al. Neurofibromin protein loss in desmoplastic melanoma subtypes: implicating NF1 allelic loss as a distinct genetic driver? Hum Pathol 2016;53:82-90.

9 Yang S, Leone D, Frydenlund N, et al. Frequency of telomerase reverse transcripter promoter mutations in desmoplastic melanoma subtypes: analyses of 76 cases. Melanoma Res 2016;26:361-366.

10 Jahn SW, Kashofer K, Halbwedl I, et al. Mutational dichotomy in desmoplastic malignant melanoma corroborated by multigene panel analysis. Mod Pathol 2015;28:895-903.

11 Francisco LM, Sage PT, Sharpe AH. The PD-1 pathway in tolerance and autoimmunity. Immunol Rev 2010;236:219-242.

12 Llosa NJ, Cruise M, Tam A, et al. The vigorous immune microenvironment of microsatellite instable colon cancer is balanced by multiple counter-inhibitory checkpoints. Cancer Discov 2015;5:43-51.

13 Robert C, Long GV, Brady B, et al. Nivolumab in previously untreated melanoma without BRAF mutation. N Engl J Med 2015;372:320-330.

14 Topalian SL, Hodi FS, Brahmer JR, et al. Safety, activity, and immune correlates of anti-PD-1 antibody in cancer. N Engl J Med 2012;366:2443-2454.

15 Robert C, Schachter J, Long GV, et al. Pembrolizumab versus ipilimumab in advanced melanoma. N Engl J Med 2015;372:2521-2532.

16 Herbst RS, Soria J-C, Kowanetz M, et al. Predictive correlates of response to the anti-PD-L1 antibody MPDL3280A in cancer patients. Nature 2014;515: 563-567.

17 Tumeh PC, Harview CL, Yearley JH, et al. PD-1 blockade induces responses by inhibiting adaptive immune resistance. Nature 2014;515:568-571.

18 Taube JM, Klein A, Brahmer JR, et al. Association of PD-1, PD-1 ligands, and other features of the tumor immune microenvironment with response to anti-PD-1 therapy. Am Assoc Cancer Res 2014;20:5064-5074.

19 Eroglu Z, Kim DW, Johnson DB, et al. Response to anti-PD1/PDL1 therapy in patients with metastatic desmoplastic melanoma. ASCO Meet Abstr 2015;33: 9011.

20 Rodić N, Anders RA, Eshleman JR, et al. PD-L1 expression in melanocytic lesions does not correlate with the BRAF V600E mutation. Cancer Immunol Res 2015;3:110-115.

21 Shain AH, Garrido M, Botton T, et al. Exome sequencing of desmoplastic melanoma identifies recurrent NFKBIE promoter mutations and diverse activating mutations in the MAPK pathway. Nat Genet 2015;47: 1194-1199. 
22 Rabbie R, Adams DJ. Desmoplastic melanoma: C > Ts and NF-кB. Pigment Cell Melanoma Res 2016;29:120-121.

23 Snyder A, Makarov V, Merghoub T, et al. genetic basis for clinical response to CTLA-4 blockade in melanoma. N Engl J Med 2014;371:2189-2199.

24 Allen EMV, Miao D, Schilling B, et al. Genomic correlates of response to CTLA-4 blockade in metastatic melanoma. Science 2015;350:207-211.

25 Madore J, Strbenac D, Vilain R, et al. PD-L1 negative status is associated with lower mutation burden, differential expression of immune-related genes, and worse survival in stage III melanoma. Am Assoc Cancer Res 2016;22:3915-3923.

26 Kakavand H, Vilain RE, Wilmott JS, et al. Tumor PD-L1 expression, immune cell correlates and PD-1+ lymphocytes in sentinel lymph node melanoma metastases. Mod Pathol 2015;28:1535-1544.

27 Madore J, Vilain RE, Menzies AM, et al. PD-L1 expression in melanoma shows marked heterogeneity within and between patients: implications for antiPD-1/PD-L1 clinical trials. Pigment Cell Melanoma Res 2015;28:245-253.

28 Thierauf J, Veit JA, Affolter A, et al. Identification and clinical relevance of PD-L1 expression in primary mucosal malignant melanoma of the head and neck. Melanoma Res 2015;25:503-509.

29 Taube JM, Anders RA, Young GD, et al. Colocalization of inflammatory response with B7-H1 expression in human melanocytic lesions supports an adaptive resistance mechanism of immune escape. Sci Transl Med 2012;4:127ra37-127ra37.

30 Hino R, Kabashima K, Kato $\mathrm{Y}$, et al. Tumor cell expression of programmed cell death-1 ligand 1 is a prognostic factor for malignant melanoma. Cancer 2010;116:1757-1766.

31 Massi D, Brusa D, Merelli B, et al. The status of PD-L1 and tumor-infiltrating immune cells predict resistance and poor prognosis in BRAFi-treated melanoma patients harboring mutant BRAFV600. Ann Oncol 201526: 1980-1987.

32 Massi D, Brusa D, Merelli B, et al. PD-L1 marks a subset of melanomas with a shorter overall survival and distinct genetic and morphological characteristics. Ann Oncol 2014;25:2433-2442.

33 Frydenlund N, Mahalingam M. Desmoplastic melanoma, neurotropism, and neurotrophin receptors-what we know and what we do not. Adv Anat Pathol 2015;22:227-241.

34 Larkin J, Hodi FS, Wolchok JD. Combined Nivolumab and Ipilimumab or monotherapy in untreated melanoma. N Engl J Med 2015;373:1270-1271.

35 Weber JS, Kudchadkar RR, Yu B, et al. Safety, efficacy, and biomarkers of Nivolumab with vaccine in Ipilimumab-refractory or -naive melanoma. J Clin Oncol 2013;31:4311-4318.

36 Latchman Y, Wood CR, Chernova T, et al. PD-L2 is a second ligand for PD-1 and inhibits $\mathrm{T}$ cell activation. Nat Immunol 2001;2:261-268. 